(1)

CrossMark

\title{
COVID-19 phenotypes: leading or misleading?
}

To the Editor:

We read the editorial by Bos et al. [1] with a mixture of interest, irritation and serious concern.

Our interest derives from a simple fact: the debate on terms like "typical acute respiratory distress syndrome (ARDS)" or "atypical ARDS" is not just a question of semantics; these terms represent concepts linked to specific clinical, mechanical and radiological criteria, and are not merely based on the severity of gas exchange. It should not be a surprise to the authors that different radiological patterns and mechanical characteristics should suggest different ventilatory strategies, each with possible benefits and harm. The management of individual patients needs to take into consideration various factors, and not just the gas exchange that currently defines ARDS. This is precisely the point of bringing attention to the novel "L" and " $\mathrm{H}$ " phenotypes of coronavirus disease 2019 (COVID-19) that bracket the extremes of the clinical encounter [2]. Usually, there is overlap, depending in large part on disease duration. The " $L$ " and " $H$ " were not intended to be tightly prescriptive nor mutually exclusive "bins" into which each patient falls, as we clearly stated previously [3]. Rather, the object was to alert clinicians, in order to avert potential harm from assuming usual ARDS associations between hypoxaemia and mechanics at all stages. In so doing, we hoped to help prevent use of high positive end-expiratory pressure when there is no benefit and, equally important, to avoid maintaining low pressures when higher pressures can be beneficial.

The irritation derives from the fact that Bos et al. [1] seem to have deliberately decided to ignore the pathophysiological "evidence" readily available and ventured into a philosophical and semantic discourse against "premature phenotyping", and in so doing committing the greater sin of "premature adjudication". After reading sentences such as "By needlessly clouding the clinical picture, false phenotypes [...] upon inspection of patient data, simply do not exist", it is not clear to us (and without a doubt to most readers) what sort of clear and self-evident truth we (and other authors) have been trying to cloud. The fact that COVID-19 patients with similar oxygenation efficiency may have markedly different compliance (and risk of ventilator-induced lung injury) is apparent to any clinician who has ever looked after a number of these patients. The reasoning put forward by the editorialists seems purely argumentative and inflammatory, as it seems to imply that what we propose is based on non-existent data, i.e. a perception that we invented.

Our concern derives from noting that the observations of Bos et al. [1] are expressed with a tone that goes beyond healthy and reasonable scientific debate. We note also with concern the conclusions of the editorial: "By prematurely phenotyping patients with COVID-19, we expose ourselves and our patients to considerable and preventable risk" and we invite the authors to express with clarity the risks they are referring to and how their argument is furthering the cause of patients and clinicians. Time and emerging literature will undoubtedly demonstrate where "truth" lies.

Luciano Gattinoni $\odot^{1}$, Luigi Camporota ${ }^{2}$ and John J. Marini ${ }^{3}$

${ }^{1}$ Dept of Anesthesiology, Emergency and Intensive Care Medicine, University of Göttingen, Göttingen, Germany. ${ }^{2}$ Dept of Adult Critical Care, Guy's and St Thomas' NHS Foundation Trust, King's Health Partners, and Division of Asthma, Allergy and Lung Biology, King's College London, London, UK. ${ }^{3}$ Regions Hospital and University of Minnesota, St Paul, MN, USA.

Correspondence: Luciano Gattinoni, University of Göttingen, Dept of Anesthesiology, Robert-Koch-Straße 40, 37075 Göttingen, Germany. E-mail: gattinoniluciano@gmail.com

Received: 6 June 2020 | Accepted after revision: 11 June 2020

@ERSpublications

Comment on the editorial by Bos et al. about "perils of premature phenotyping in COVID-19", querying the risks of COVID-19 phenotypes and how the arguments put forward can further the cause of patients and clinicians https://bit.ly/2Y6VZz9

Cite this article as: Gattinoni L, Camporota L, Marini JJ. COVID-19 phenotypes: leading or misleading? Eur Respir J 2020; 56: 2002195 [https://doi.org/10.1183/13993003.02195-2020]. 
Conflict of interest: None declared.

\section{References}

1 Bos LDJ, Sinha P, Dickson RP. The perils of premature phenotyping in COVID-19: a call for caution. Eur Respir J 2020; 56: 2001768.

2 Gattinoni L, Chiumello D, Caironi P, et al. COVID-19 pneumonia: different respiratory treatments for different phenotypes? Intensive Care Med 2020; 46: 1099-1102.

3 Marini JJ, Gattinoni L. Management of COVID-19 respiratory distress. JAMA 2020; 323: 2329-2330.

\section{Building the house of CARDS by phenotyping on the fly}

To the Editor:

Some patients with coronavirus disease 2019 (COVID-19), fulfilling the Berlin criteria for acute respiratory distress syndrome (ARDS), do not respond well to the current treatment paradigm [1]. The perspective by RELLo et al. [2] on phenotypes of COVID-19, and the editorial by Bos et al. [3], are therefore of great interest. The "responsible" phenotyping of COVID-19 ARDS (CARDS) recommended by Bos et al. [3] may be expedited by re-evaluating the existing literature on refractory hypoxia.

In 2000, the landmark ARDS Network (ARDSNet) trial demonstrated that ventilation with low tidal volumes $\left(V_{\mathrm{T}} ; 6-8 \mathrm{~mL} \cdot \mathrm{kg}^{-1}\right.$ predicted body weight $\left.(\mathrm{PBW})\right)$, titration of positive end-expiratory pressure (PEEP) to inspiratory oxygen fraction and maintaining plateau pressure under $30 \mathrm{cmH}_{2} \mathrm{O}$ significantly reduced mortality [4]. The mortality in the group that received ARDSNet ventilation (31.0\%) was significantly lower than that of the control group (39.8\%) who were ventilated with a "traditional" high $V_{\mathrm{T}}$ strategy (12 mL·kg $\left.{ }^{-1} \mathrm{PBW}\right)$ [4]. Absolute risk reduction was $8.8 \%$, so the number needed to treat (NNT) to prevent one death is 11.4 .

So, for the past 20 years, the ARDSNet protocol has set the standard for ventilation of patients with ARDS. Bos et al. [3] essentially say that this is rightly so, and suggest that the ARDSNet protocol should also be rigorously applied to CARDS. Indeed, the Surviving Sepsis Campaign guidelines for the management of COVID-19 [5] support Bos et al. [3].

However, in the ARDSNet trial, approximately 30\% of patients receiving ARDSNet ventilation died, and just over $60 \%$ of controls survived [4]. Thus, although the NNT is low, of every 11.4 patients with ARDS, 10.4 do not benefit from this ventilatory strategy and $60 \%$ can tolerate high $V_{\mathrm{T}}$.

In 2015, Амато et al. [6] reported a multilevel mediation reanalysis of pooled data from four randomised controlled trials of ventilatory strategies for ARDS. This showed that driving pressure (i.e. plateau pressure minus total PEEP; $\triangle \mathrm{P}$ ) was the ventilator variable most strongly associated with survival. Any change in $V_{\mathrm{T}}$ or PEEP only improved outcomes if associated with a fall in $\Delta \mathrm{P}[6]$.

Thus, while the net effect of the ARDSNet protocol is beneficial at the level of the study population, theoretically it may harm select patients, particularly when not associated with a fall in $\Delta \mathrm{P}$. Therefore, contrary to the opinions of the Surviving Sepsis Campaign [5] and Bos et al. [3], the ARDSNet protocol is not a panacea. Unfortunately, the subgroup of patients with ARDS who do not benefit from the ARDSNet protocol is a "known unknown". So individualising ventilatory support is currently extremely challenging.

@ERSpublications

Using clinical, pathophysiological and immunological phenotyping of ARDS to refine management of COVID-19 is urgently required to improve outcomes from refractory hypoxia https://bit.ly/2VvZelp

Cite this article as: Rajendram R. Building the house of CARDS by phenotyping on the fly Eur Respir J 2020; 56: 2002429 [https://doi.org/10.1183/13993003.02429-2020]. 
To improve outcomes, further research is required to determine which patients benefit from the ARDSNet protocol (i.e. phenotyping). This will allow consideration of alternative strategies for patients who are unlikely to benefit from the ARDSNet protocol.

Sadly, the literature on ARDS is littered with promising interventions that were associated with improved outcomes in case reports, case series and observational studies but were subsequently discarded after large randomised controlled trials. This may reflect the shortcomings of previous research on ARDS. Indiscriminate recruitment of heterogeneous cohorts of patients generated significant noise, which may have drowned out any potential benefits in specific subgroups of patients.

The COVID-19 pandemic provides the unique opportunity to rectify this deplorable situation by responsibly phenotyping "on the fly". The evidence-base for the management of refractory hypoxia could be significantly advanced by analysing the effect of interventions such as nitric oxide and prone positioning on multiple phenotypes of ARDS with a unique aetiology. Observations in CARDS may be relevant to other respiratory diseases. However, to increase generalisability, future studies should, a priori, explore outcomes in clinically, pathophysiologically and immunologically defined subgroups.

\section{Rajkumar Rajendram $\oplus^{1,2}$}

${ }^{1}$ Dept of Medicine, King Abdulaziz Medical City, King Abdulaziz International Medical Research Center, Ministry of National Guard - Health Affairs, Riyadh, Saudi Arabia. ${ }^{2}$ College of Medicine, King Saud bin Abdulaziz University of Health Sciences, Riyadh, Saudi Arabia.

Correspondence: Rajkumar Rajendram, Dept of Medicine, King Abdulaziz Medical City, Ministry of National Guard Health Affairs, Riyadh, Saudi Arabia. E-mail: rajkumarrajendram@doctors.org.uk

Received: 21 June 2020 | Accepted: 28 June 2020

Author contributions: R. Rajendram developed the hypothesis, performed the literature search and refined the hypothesis. R. Rajendram prepared the initial draft of the manuscript, revised the manuscript and approved the final version of the manuscript for publication.

Conflict of interest: None declared.

\section{References}

1 Gattinoni L, Coppola S, Cressoni M, et al. COVID-19 does not lead to a "typical" acute respiratory distress syndrome. Am J Respir Crit Care Med 2020; 201: 1299-1300.

2 Rello J, Storti E, Belliato M, et al. Clinical phenotypes of SARS-CoV-2: implications for clinicians and researchers. Eur Respir J 2020; 55: 2001028.

3 Bos LDJ, Sinha P, Dickson RP. The perils of premature phenotyping in COVID-19: a call for caution. Eur Respir J 2020; 56: 2001768.

4 Brower RG, Matthay MA, Morris A, et al. Ventilation with lower tidal volumes as compared with traditional tidal volumes for acute lung injury and the acute respiratory distress syndrome. N Engl J Med 2000; 342: 1301-1308.

5 Alhazzani W, Møller MH, Arabi YM, et al. Surviving Sepsis Campaign: guidelines on the management of critically ill adults with coronavirus disease 2019 (COVID-19). Crit Care Med 2020; 48: e440-e469.

6 Amato MBP, Meade MO, Slutsky AS, et al. Driving pressure and survival in the acute respiratory distress syndrome. N Engl J Med 2015; 372: 747-755. 


\section{COVID-19 conundrum: clinical phenotyping based on pathophysiology as a promising approach to guide therapy in a novel illness}

To the Editor:

We read with interest the recent editorial by Bos et al. [1] on the perils of premature phenotyping in coronavirus disease 2019 (COVID-19). The authors concluded that a normal compliance variant of acute respiratory distress syndrome (ARDS) does not exist, based on two small cohort studies reporting low respiratory system compliance in COVID-19 patients $[2,3]$. However, this assumption may be erroneous, as, first, the admission and intubation thresholds are highly variable across units, resulting in marked heterogeneity. Secondly, several studies demonstrate that a high proportion of mechanically ventilated COVID-19 patients exhibit near-normal lung compliance [4-6].

These observations, on first glance, seem incompatible with the current understanding of ARDS pathophysiology, as profound hypoxaemia and normal lung compliance rarely co-exist in ARDS [7]. A heuristic approach would be to ignore these inconsistencies, attempting to "fit" them into existing paradigms. However, initial intuitions may often be wrong, and cognitive biases must be overcome to find a solution to this conundrum. Using a deductive approach, firstly the diagnostic criteria need a relook, to exclude misclassification as a reason for the observed clinico-pathological discrepancy.

ARDS is characterised by diffuse alveolar damage, with increased pulmonary vascular permeability, loss of aerated lung tissue and low respiratory system compliance [8]. However, several unrelated pathologies such as eosinophilic pneumonia or diffuse alveolar haemorrhage may cause respiratory failure fulfilling the clinical criteria for ARDS [9]. Accordingly, these "ARDS mimics" [9] require specific treatment based on their underlying pathophysiology.

Several other conditions presenting with hypoxaemia and normal lung compliance may additionally be misclassified as ARDS, diffuse microvascular pulmonary thrombosis being one such pathology. In a case report [10], the clinical presentation was "ARDS-like", with profound hypoxaemia and bilateral infiltrates on radiology, but with normal ventilatory parameters on spirometry. Such disorders, where perfusion impairment is the dominant mechanism for hypoxaemia, cannot be considered as "true" ARDS [6]. This lack of diagnostic specificity of the Berlin definition for underlying pathology could be due to the omission of objective indicators of lung volume loss, such as low respiratory system compliance, in its final version [8].

Emerging evidence indicates in situ thrombosis leading to perfusion loss as the dominant initial pathology in COVID-19 lung injury. The early radiological changes of ground-glassing and consolidation in COVID-19 were considered to be infective or inflammatory in aetiology [11]. However, recent paired parenchymal-perfusion imaging studies demonstrate well-demarcated perfusion defects underlying these changes, implicating a thrombotic aetiology [4, 12-16]. Unmatched defects are also seen $[4,15]$. Moreover, the parenchymal changes follow a peripheral "vascular distribution", which are often wedge-shaped [11, 16]. These findings suggest that the primary insult is vaso-occlusive, as infections or inflammation are rarely confined to vascular boundaries. Additionally, proximal vascular dilatation suggests distal vessel occlusion [13, 16]. Interestingly, rapid radiological resolution and clinical improvement with inhaled thrombolytics have been described in a small case series [17].

Autopsy findings of viral endotheliitis further clarify the pathogenesis of thrombotic manifestations in COVID-19 [18, 19], with a prothrombotic cytokine response [20] that mirrors the response seen in extensive

@ERSpublications

The current evidence of COVID-19 pathophysiology supports the idea of specific phenotypes, and clinical phenotyping may be valuable to guide therapy https://bit.ly/2ZagmMn

Cite this article as: Cherian R, Chandra B, Tung ML, et al. COVID-19 conundrum: clinical phenotyping based on pathophysiology as a promising approach to guide therapy in a novel illness Eur Respir J 2020; 56: 2002135 [https://doi.org/10.1183/13993003.02135-2020]. 


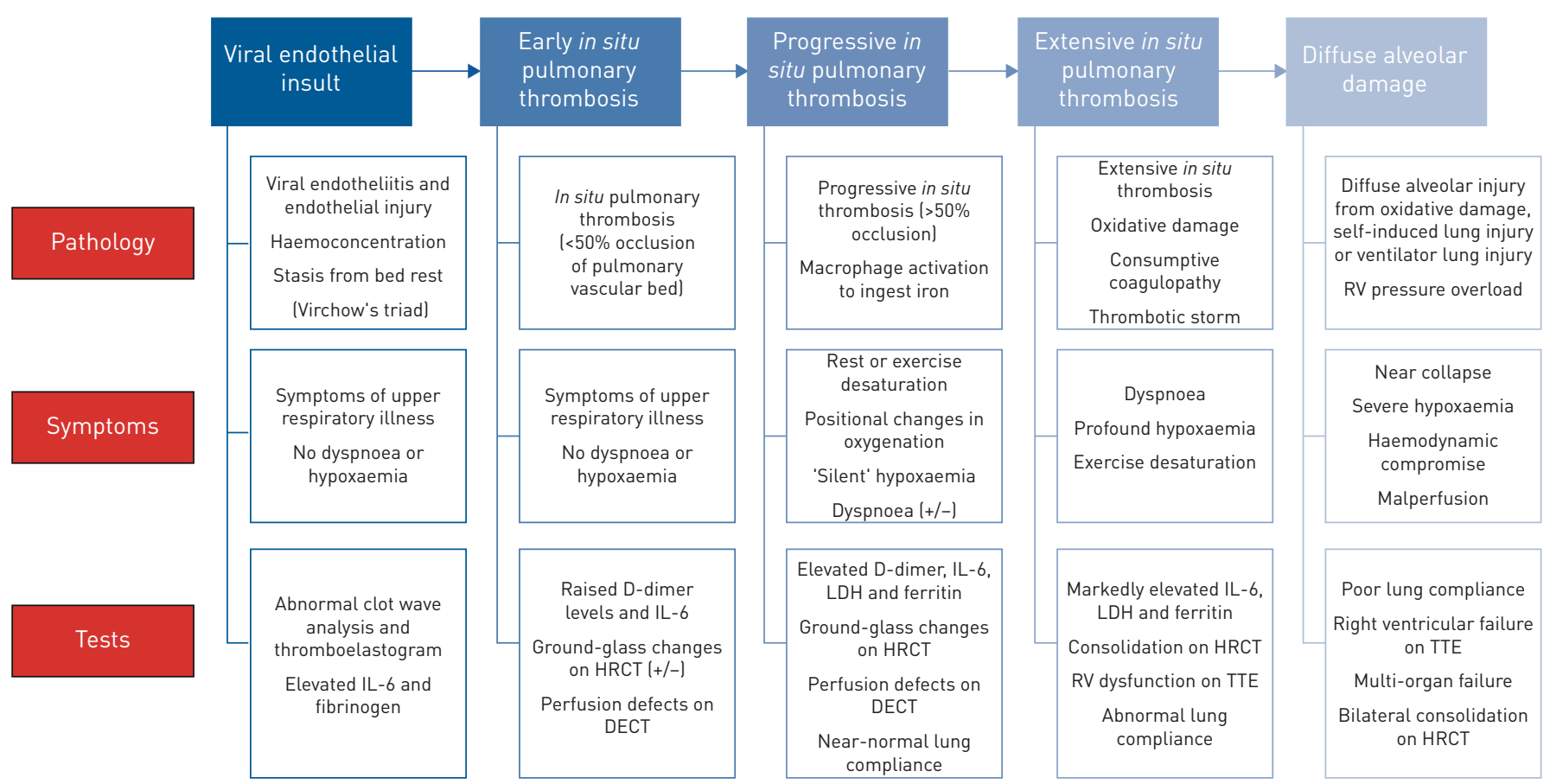

FIGURE 1: Progression of COVID-19-related lung injury and respiratory failure. Viraemia with viral endotheliitis fuels an inflammatory response appropriate for vascular injury, resulting in a prothrombotic state. Interleukin (IL)-6 upregulates fibrinogen gene expression. Pulmonary in situ thrombosis is facilitated by Virchow's triad. Early disease is subclinical due to lung perfusion reserve. Progression may be aborted in young individuals with rapid endothelial turnover and robust intrinsic thrombolysis. Progressive in situ microvascular thrombosis eventually leads to hypoxaemia when reserves are exhausted. Initial hypoxaemia may be silent (no dyspnoea) as lung compliance is normal. Oxidative damage from iron and haem in the presence of unextracted alveolar oxygen, after perfusion loss, may be a major determinant of parenchymal injury. Additionally, self-induced lung injury, ventilator lung injury and secondary infections result in diffuse alveolar damage. D-dimer, lactate dehydrogenase (LDH) and ferritin are elevated sequentially. Pulmonary in situ thrombosis as the initial insult and major determinant of COVID-19-related lung injury explains the observed clinical phenotypes and disease spectrum. Early risk stratification and anticoagulation may avert thrombotic storm. RV: right ventricle; HRCT: high-resolution computed tomography; DECT: dual-energy computed tomography; TTE: transthoracic echocardiogram.

vascular injury [21]. Furthermore, natural and iatrogenic sequelae could explain the observed phenotypic heterogeneity of COVID-19 (figure 1) [5, 7]. It is of note that diffuse alveolar damage is not universally found on autopsies [22], suggesting this as a sequela and the terminal pathology rather than the index event. Conversely, diffuse pulmonary microthrombosis is consistently visible on autopsies [18, 22, 23].

Additionally, clinical observations indicate perfusion loss as the primary mechanism of hypoxaemia in early COVID-19 respiratory failure. The early COVID-19 respiratory failure phenotype, with hypoxaemia in the presence of preserved lung mechanics [4-6], suggests perfusion impairments as the main pathophysiology. Although the ground-glass changes signify ventilatory impairments, owing to perfusion loss, the affected alveoli act as dead spaces rather than shunts. In this situation, hypoxaemia occurs primarily due to flow redistribution and overperfusion involving a significantly reduced vascular bed, resulting in ventilation/perfusion mismatch [24, 25]. This typically requires loss of $40-50 \%$ of the pulmonary vascular bed before clinically significant hypoxaemia could occur, indicating a large reserve. Reduced mixed venous saturation could further exacerbate hypoxaemia. Also, during extensive obstruction, available time for red cell oxygenation within the alveolar capillaries may be reduced, due to insufficient microcirculatory recruitment and increased flow velocities. Diffusion limitation may result, further aggravating hypoxaemia $[24,25]$.

Evidently, the clinical phenotype of perfusion loss differs markedly from that of primary alveolar disease. While hypoxaemia can result either from ventilatory impairments or from disorders of alveolar perfusion, there are stark differences in their clinical features. In alveolar diseases such as pneumonia, shunt perfusion results in early hypoxaemia, with clinically proportionate dyspnoea due to abnormal lung compliance. However, in progressive perfusion loss, hypoxaemia manifests late due to the large lung perfusion reserve, initial ventilatory compensation that mitigates ventilation/perfusion mismatch from overperfusion, and adequate initial right ventricular compensation to acute pressure overload. Furthermore, unlike ventilatory disorders, the initial hypoxaemia in this situation may be "silent", owing to minimal parenchymal injury and normal lung compliance at this stage. However, once dyspnoea sets in, 
there would be rapid clinical progression whereby minor changes in mixed venous saturations or transit time could result in major changes in systemic oxygen saturation. Correspondingly, as the perfusion reserve continues to decline, physiological stress and exertion would be poorly tolerated. Eventually, right heart compromise would result in cardiorespiratory collapse and rapidly progressing multi-organ failure. These insights are key to understanding the clinical phenotypes of COVID-19 lung injury.

In summary, although COVID-19 respiratory failure may fulfil the Berlin criteria, it would be inappropriate to describe the early lung pathology of progressive pulmonary in situ thrombosis as ARDS. Evidence-based therapies for ARDS may not be applicable at this stage of illness, as the lung mechanics and haemodynamics mirror that of a large pulmonary embolism. Moreover, a protocol-based "one size fits all" approach could potentially be catastrophic, as employing a high positive end-expiratory pressure strategy in a normally compliant lung would result in significant barotrauma and deterioration of right heart dysfunction. As lung pathology appears grossly different at various stages of illness, a tailored phenotypic approach to management, guided by pathophysiology, would be more appropriate than a syndromic approach.

Robin Cherian $\oplus^{1}$, Bharatendu Chandra ${ }^{2}$, Moon Ley Tung $\oplus^{3}$ and Alain Vuylsteke $\oplus^{4}$

${ }^{1}$ Dept of Cardiology, National University Heart Centre Singapore, Singapore, Singapore. ${ }^{2}$ Division of Neurology, National University Hospital, Singapore, Singapore. ${ }^{3}$ Dept of Haematology-Oncology, National University Cancer Institute, Singapore, Singapore. ${ }^{4}$ Dept of Anaesthesia and Critical care, Royal Papworth Hospital NHS Trust, Cambridge, UK.

Correspondence: Robin Cherian, Cardiac Critical Care, Dept of Cardiology, National University Heart Centre, NUHS Tower Block, Level 9, 1E Kent Ridge Road, Singapore 119228. E-mail: robin_cherian@nuhs.edu.sg

Received: 3 June 2020 | Accepted after revision: 3 July 2020

Conflict of interest: None declared.

\section{References}

1 Bos LDJ, Sinha P, Dickson RP. The perils of premature phenotyping in COVID-19: a call for caution. Eur Respir J 2020; 56: 2001768.

2 Ziehr DR, Alladina J, Petri CR, et al. Respiratory pathophysiology of mechanically ventilated patients with COVID-19: a cohort study. Am J Respir Crit Care Med 2020; 201: 1560-1564.

3 Bhatraju PK, Ghassemieh BJ, Nichols M, et al. COVID-19 in critically ill patients in the Seattle region - case series. N Engl J Med 2020; 382: 2012-2022.

4 Mauri T, Spinelli E, Scotti E, et al. Potential for lung recruitment and ventilation-perfusion mismatch in patients with the acute respiratory distress syndrome from coronavirus disease 2019. Crit Care Med 2020; 48: 1129-1134.

5 Rello J, Storti E, Belliato M, et al. Clinical phenotypes of SARS-CoV-2: implications for clinicians and researchers. Eur Respir J 2020; 55: 2001028.

6 Gattinoni L, Coppola S, Cressoni M, et al. COVID-19 does not lead to a "typical" acute respiratory distress syndrome. Am J Respir Crit Care Med 2020; 201: 1299-1300.

7 Gattinoni L, Chiumello D, Caironi P, et al. COVID-19 pneumonia: different respiratory treatments for different phenotypes? Intensive Care Med 2020; 46: 1099-1102.

8 ARDS Definition Task Force, Ranieri VM, Rubenfeld GD, et al. Acute respiratory distress syndrome: the Berlin Definition. JAMA 2012; 307: 2526-2533.

9 Matthay MA, Zemans RL, Zimmerman GA, et al. Acute respiratory distress syndrome. Nat Rev Dis Primers 2019; 5: 18.

10 Maggiorini M, Knoblauch A, Schneider J, et al. Diffuse microvascular pulmonary thrombosis associated with primary antiphospholipid antibody syndrome. Eur Respir J 1997; 10: 727-730.

11 Ye Z, Zhang Y, Wang Y, et al. Chest CT manifestations of new coronavirus disease 2019 (COVID-19): a pictorial review. Eur Radiol 2020; 30: 4381-4389.

12 Tee A, Wong A, Yusuf GT, et al. Contrast-enhanced ultrasound (CEUS) of the lung reveals multiple areas of microthrombi in a COVID-19 patient. Intensive Care Med 2020; 46: 1660-1662.

13 Lang M, Som A, Mendoza DP, et al. Hypoxaemia related to COVID-19: vascular and perfusion abnormalities on dual-energy CT. Lancet Infect Dis 2020; in press [https://doi.org/10.1016/S1473-3099(20)30367-4].

14 Soldati G, Giannasi G, Smargiassi A, et al. Contrast-enhanced ultrasound in patients with COVID -19: pneumonia, acute respiratory distress syndrome, or something else? J Ultrasound Med 2020; in press [https://doi. org/10.1002/jum.15338]

15 Sciagrà R, Rubino F, Malandrino $\mathrm{D}$, et al. Are disease-related pulmonary perfusion abnormalities detectable in COVID-19 patients? Suspicious findings in a lung perfusion SPECT performed for ruling out classical pulmonary embolism. Eur J Nucl Med Mol Imaging 2020; 47: 2211-2213.

16 Oudkerk M, Büller HR, Kuijpers D, et al. Diagnosis, prevention, and treatment of thromboembolic complications in COVID-19: report of the National Institute for Public Health of the Netherlands. Radiology 2020; in press [https://doi.org/10.1148/radiol.2020201629].

17 Wu Y, Wang T, Guo C, et al. Plasminogen improves lung lesions and hypoxemia in patients with COVID-19. QJM 2020; 113: 539-545.

18 Ackermann M, Verleden SE, Kuehnel M, et al. Pulmonary vascular endothelialitis, thrombosis, and angiogenesis in COVID-19. N Engl J Med 2020; 383: 120-128.

19 Varga Z, Flammer AJ, Steiger P, et al. Endothelial cell infection and endotheliitis in COVID-19. Lancet 2020; 395: 1417-1418. 
20 McGonagle D, O’Donnell JS, Sharif K, et al. Immune mechanisms of pulmonary intravascular coagulopathy in COVID-19 pneumonia. Lancet Rheumatol 2020; 2: E437-E445.

21 Shenkar R, Coulson WF, Abraham E. Hemorrhage and resuscitation induce alterations in cytokine expression and the development of acute lung injury. Am J Respir Cell Mol Biol 1994; 10: 290-297.

22 Wichmann D, Sperhake JP, Lütgehetmann M, et al. Autopsy findings and venous thromboembolism in patients with COVID-19. Ann Intern Med 2020; in press [https://doi.org/10.7326/M20-2003].

23 Lax SF, Skok K, Zechner P, et al. Pulmonary arterial thrombosis in COVID-19 with fatal outcome: results from a prospective, single-center, clinicopathologic case series. Ann Intern Med 2020; in press [https://doi.org/10.7326/ M20-2566].

24 Petersson J, Glenny RW. Gas exchange and ventilation-perfusion relationships in the lung. Eur Respir J 2014; 44: 1023-1041.

25 Wagner PD. The physiological basis of pulmonary gas exchange: implications for clinical interpretation of arterial blood gases. Eur Respir J 2015; 45: 227-243.

\section{Response to COVID-19 phenotyping correspondence}

From the authors:

In their letter, R. Cherian and co-workers take issue with our interpretation of the respiratory physiology of coronavirus disease 2019 (COVID-19), arguing that it is based merely on "small cohort studies", and instead declaring that "a high proportion of mechanically ventilated COVID-19 patients exhibit near-normal lung compliance”. Yet the low respiratory compliance of COVID-19 patients has now been extensively demonstrated by studies totalling more than 800 COVID-19 patients [1-7], including a direct comparison with non-COVID-19 acute respiratory distress syndrome (ARDS) patients that revealed no difference in respiratory compliance [7]. In contrast, the three case series cited by R. Cherian and co-workers in support of their claim comprise cohorts of, respectively, 16, 10 and 26 patients [8-10]. Furthermore, even these case series report average respiratory compliance in COVID-19 of 40-45 $\mathrm{mL} \cdot \mathrm{cm} \mathrm{H}_{2} \mathrm{O}^{-1}$, which is in fact abnormal and far from "near-normal compliance" [11, 12]. As an informative comparison, the ANZICS (Australian and New Zealand Intensive Care Society) cohort of ARDS patients used to derive the Berlin definition of ARDS had an average respiratory compliance of $40 \pm 15 \mathrm{~mL} \cdot \mathrm{cmH}_{2} \mathrm{O}^{-1}$ [13]. We thus find no evidence in the authors' citations (or elsewhere) to support their empirical claim that many or most COVID-19 patients present with "normal" or "near-normal" respiratory compliance.

R. Cherian and co-workers also assume a temporal progression from "early" COVID-19 physiology (characterised by normal respiratory compliance) to "late" physiology (characterised by impaired respiratory compliance). Yet three published studies comprising nearly 350 mechanically ventilated COVID-19 patients have reported serial measurements of respiratory compliance $[1,3,6]$, and none has shown any temporal trend towards decreased compliance in the days following initiation of mechanical ventilation. Furthermore, a recent report from HaUdEBourg et al. [7] demonstrated no correlation between duration of symptoms and respiratory compliance in COVID-19 patients (figure 1a). We have since validated this observation using our own clinical data (figure 1b). As shown in figure 1c, GatTINONI et al. [14] recently published their own data countering these findings. Importantly, when data from all three cohorts are combined and analysed together, no temporal trend is present $\left(p=0.50, r^{2}=0.005\right.$; figure 1e). Closer inspection reveals that the purported correlation in the cohort of GATTINONiet al. [14] is entirely attributable to two patients with low respiratory compliance and $>3$ weeks of symptoms, a duration of

@ERSpublications

Phenotyping of COVID-19-related ARDS should be done using careful, data-driven approaches https://bit.ly/3eX65Yu

Cite this article as: Bos LDJ, Sinha P, Dickson RP. Response to COVID-19 phenotyping correspondence Eur Respir J 2020; 56: 2002756 [https://doi.org/10.1183/13993003.02756-2020]. 
disease irrelevant to considerations of acute pathogenesis and rarely encountered in the literature and not at all by Haudebourg et al. [7] or ourselves (figure 1a and b). As shown in figure 1d, if analysis is restricted to the 35 patients with symptoms shorter than 3 weeks, there is no evidence of a temporal correlation ( $\left.\mathrm{p}=0.72, \mathrm{r}^{2}=0.004\right)$, and the results align with the meta-analysis (figure 1e).

Taken together, the two central observations undergirding the proposed pathophysiological framework of R. Cherian and co-workers (normal respiratory mechanics in COVID-19 and their temporal deterioration) are unsupported by a burgeoning evidence base. Furthermore, these analyses are valuable working
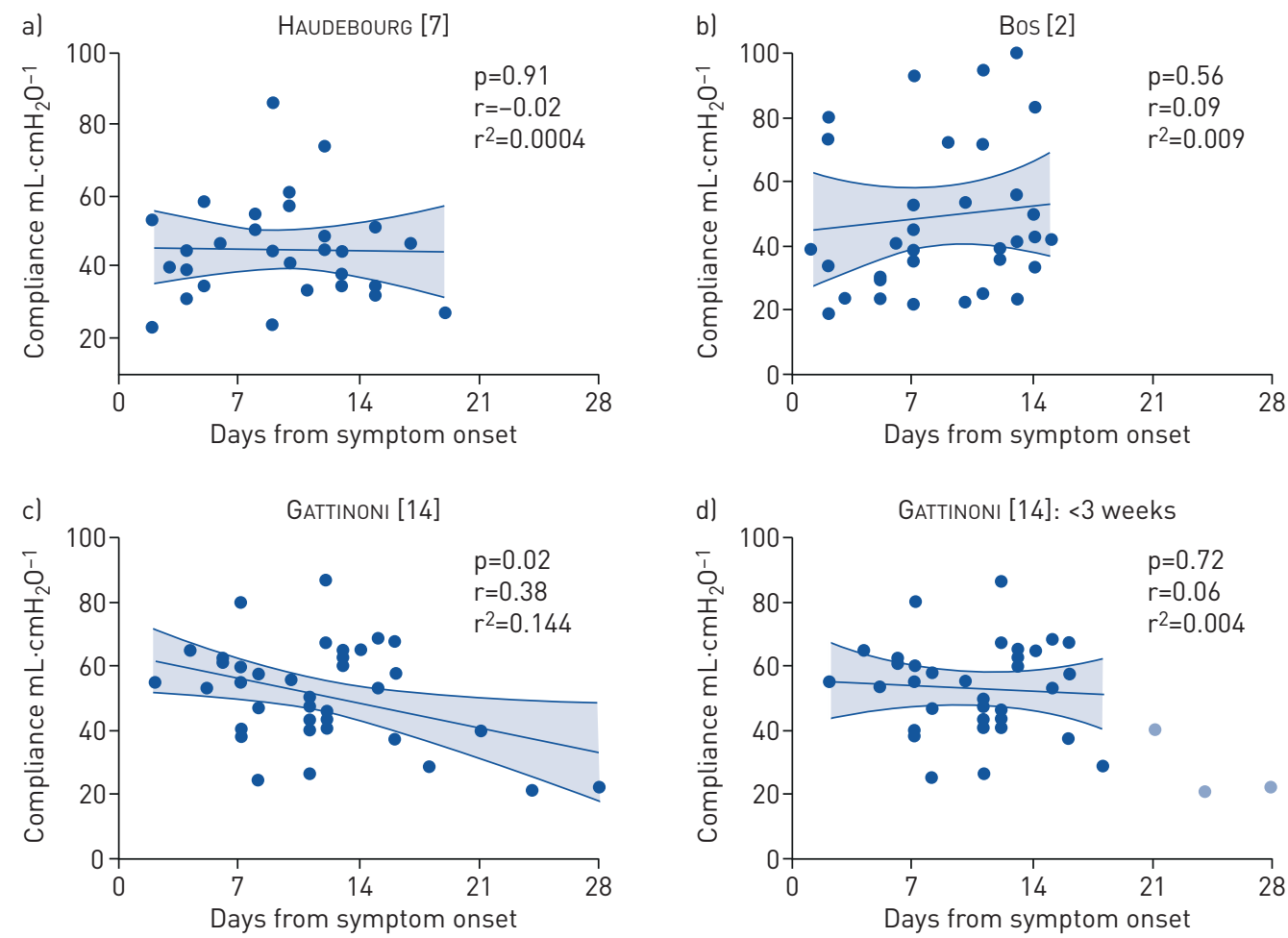

All datasets combined

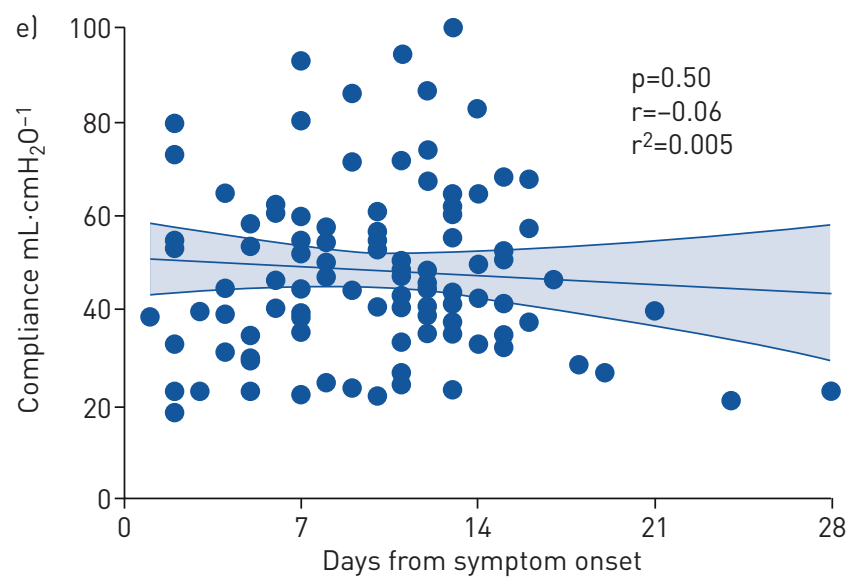

FIGURE 1 Respiratory compliance and duration of symptoms in COVID-19: a lesson in the risks of drawing premature inferences. a) HAUDEBOURG et al. [7] observed no correlation between duration of symptoms and respiratory compliance in patients with COVID-19 receiving mechanical ventilation. b) We validated this lack of association with a separate cohort [2]. c) In contrast, GATTINONI et al. [14] recently reported a negative association between duration of symptoms and respiratory compliance. d) However, this purported association was attributable entirely to three patients who had been symptomatic for 3-4 weeks la duration of questionable relevance to discussions of the pathophysiology of acute respiratory failure). e) When all datasets were combined (including the temporal outliers from GATTINONI et al. [14]], no evidence of a correlation was found. This illustrates the danger of drawing premature inferences from underpowered cohorts, which are prone to over-fitting and spurious correlations. 
demonstrations of the numerous pitfalls we cautioned against in our editorial [15]: 1) the risks of drawing premature pathophysiological conclusions from underpowered case series and then making possibly erroneous therapeutic recommendations; 2) the instability of statistical inferences using small, single-variable data sources; and 3) the predictable correction of initial human intuitions when more data emerge. A final under-appreciated and unmeasurable pitfall of premature phenotyping raised in our editorial, and one that the multitude of publications addressing these purported phenotypes are substantiating, is the cost to research resources caused by high-profile yet unsupported speculation. This factor is all the more pertinent in the face of an unforgiving pandemic in which clinical intensive care unit workload is highly demanding and clinical research is a zero-sum game. Furthermore, clinicians have even less time than usual to critically evaluate scientific literature. Therefore, it is incumbent as clinician-scientists that, whilst our data gathering may be agile and creative, its interpretation should be cautious and deliberate.

While we agree with R. Cherian and co-workers regarding the potential pathophysiological importance of endothelial injury in COVID-19, the data at hand are simply insufficient to declare if this aspect of pathogenesis is a central mediator of disease progression and lung injury in COVID-19. For example, it is worth noting that, while endothelial injury has been described in post mortem histopathological evaluations, it is not ubiquitous; epithelial injury and diffuse alveolar damage, however, are [16, 17]. Our editorial did not take a position on the pathophysiology of COVID-19, nor do we dispute the need to identify more homogenous biological pathways. Frankly, we do not believe in "typical ARDS", as the syndrome encompasses diverse aetiological pathways with only partially intersecting clinical and histopathological "bottlenecks". We are merely arguing that the currently postulated phenotypes are unconvincing, and insufficient to justify a widespread change in clinical management (as proposed by the correspondence).

In his response to our editorial [15], R. Rajendram reveals a curious misinterpretation: "Thus, while the net effect of the ARDSNet protocol is beneficial at the level of the study population, theoretically it may harm select patients [...] contrary to the opinions of the Surviving Sepsis Campaign [18], and Bos et al. [15], the ARDSNet protocol is not a panacea." Putting aside the wishful thinking of a supportive intervention functioning as a "panacea" for a condition with persistent mortality of 30-40\%, R. Rajendram (along with R. Cherian and co-workers) seems to think that we dispute the heterogeneity of ARDS, and advocate for a "one-size-fits-all" approach to its clinical management. Quite the opposite: we strongly believe that ARDS represents a pathophysiologically heterogenous syndrome and have argued the same for COVID-19-related ARDS [19]. Until well-defined biological subgroups are identified, the ceiling of effective interventions is likely to remain supportive. We also strongly suspect there are likely to be considerable biological differences between COVID-19 and non-COVID-19 ARDS [20].

Where we differ with our correspondents, we suspect, is in our lack of confidence that clinicians can identify meaningful subphenotypes using underpowered cohorts and bedside intuitions and then recommend effective interventions without testing them in a scientific study. This was the central point of our editorial and is illustrated with two examples in this response (the "normal compliance" of COVID-19 and its purported temporal worsening). As a contrast, the correspondents may consider recent pre-COVID-19 research identifying hypoinflammatory and hyperinflammatory subphenotypes in ARDS (to which we have contributed) $[8,21]$. These ARDS subphenotypes were derived using unsupervised clustering of more than 3000 rigorously adjudicated and extensively characterised patients [22]. The ARDS subphenotypes have been consistently validated across multiple cohorts and research groups [23-25]. In contrast, the high-compliance "L" phenotype, for the reasons already detailed, seems inherently unstable. Whereas it was initially described as constituting 70-80\% of COVID-19 ARDS cases [21], it now is defined as a rarely encountered extreme of a one-dimensional physiological continuum. In comparison, the previously identified ARDS subphenotypes represent distinct clinical "clusters" of patients, informed by measurements across organ systems and physiological domains in secondary analyses of well-curated cohorts of patients [22]. Yet despite this robustness, we would recommend that any therapeutic interventions for which benefits have been observed in these hyperinflammatory and hypoinflammatory phenotypes require testing in prospective trials before they are implemented into clinical practice, as they were derived using secondary analyses.

The objective of our editorial [15] was to challenge the subclassification of patients with COVID-19 that frequently occurred in the early weeks of the pandemic, based on "discussions" and "close observations", before they became entrenched dogmas. An unintended consequence of such a challenge may be that it evokes negative emotions with the reader, especially in these troubling and polarising times. We were, therefore, saddened to learn that our editorial caused irritation among L. Gattinoni and co-workers. While we vehemently disagree that "the observations of Bos et al. [15] are expressed with a tone that goes beyond healthy and reasonable scientific debate", we acknowledge that our essay was interpreted as such by 
L. Gattinoni and co-workers, and that is regrettable. We would like to clarify that the particular quoted sentences from our editorial that prompted the irritation and concern of L. Gattinoni and co-workers were aimed at premature phenotyping in general. It is an unfortunate misunderstanding that they assumed we were speaking directly and exclusively about them. For the reasons already outlined, however, we stand by our editorial.

L. Gattinoni and co-workers state that "the ' $\mathrm{L}$ ' and ' $\mathrm{H}$ ' [phenotypes] were not intended to be tightly descriptive nor mutually exclusive "bins' into which each patient falls," yet this is what is usually implied by disease "subphenotypes" or "endotypes" [26]. As described in our editorial [15], for phenotypes to be purposeful, they should be discrete, robust, generalisable, easily identifiable and, ideally, have an actionable intervention. Seemingly, almost none of these conditions are met in the current case. As an illustration, the problem with loosely defined phenotypes, as described by the correspondents, emerges when we try to precisely identify, at the bedside, who the patients are in whom they "hoped to help prevent use of high positive end-expiratory pressure when there is no benefit and, equally important, to avoid maintaining low pressures when higher pressures can be beneficial." It is difficult to conceive how these phenotypes would be identifiable using quantifiable variables and when precisely to intervene, given that L. Gattinoni and co-workers themselves concede that these phenotypes are temporally dynamic, neither mutually exclusive nor discrete, and that "usually, there is overlap".

We agree entirely with L. Gattinoni and co-workers that ventilator management should be individualised to each patient's physiology, and have never argued otherwise. In the theoretical "limit case" of a patient with normal lung compliance and minimal lung recruitability, we would similarly discourage use of high levels of positive end-expiratory pressure, as surely would most practising intensivists. We merely disagree with the conclusions of L. Gattinoni and co-workers regarding the prevalence of these theoretical patients based on data from 16 patients [21], as well as their subsequent recommendations to deviate from safe ventilatory practice for COVID-19 patients based on this limited data [27]. As catalogued already, the available data show that this purported "phenotype" is rarely encountered in COVID-19 ARDS.

Unexpectedly, L. Gattinoni and co-workers request evidence from us that their efforts at phenotyping have caused harm. Basic scientific convention, however, mandates that before they implore the field to deviate from usual practice, the burden is rather on them to demonstrate the benefits and safety of their proposed phenotyping scheme and linked interventions, using robust scientific studies. Thankfully for our patients, that is how best medical science works: primum non nocere (first, do no harm). Putting aside the complete absence of efficacy data, the validity of the physiological basis for their proposed interventions for these phenotypes has also been recently questioned [28, 29].

We hope our response clarifies for the correspondents and readers that we in no way dispute the underlying heterogeneity of ARDS, nor the uniqueness of COVID-19, nor the need for patient-tailored therapy; indeed, much of our research is focused on attaining this. We merely insist that phenotyping be done using careful, data-driven approaches. To paraphrase R. Rajendram, rather than strengthening a house of cards, we should instead aspire to build a foundation out of sturdier, more lasting materials: in this case agile, yet robust, scientific studies using a responsible, data-informed approach. At this stage of the pandemic, sufficient data points exist to equip us to advance from anecdote-based intuitions to evidence-informed science.

Lieuwe D.J. Bos $\oplus^{1,2}$, Pratik Sinha ${ }^{3,4}$ and Robert P. Dickson $\oplus^{5,6,7}$

${ }^{1}$ Intensive Care, Amsterdam University Medical Centers, location AMC, University of Amsterdam, Infection and Immunity, Amsterdam, The Netherlands. ${ }^{2}$ Dept of Respiratory Medicine, Amsterdam University Medical Centers, location AMC, University of Amsterdam, Infection and Immunity, Amsterdam, The Netherlands. ${ }^{3}$ Dept of Medicine, University of California San Francisco, San Francisco, CA, USA. ${ }^{4}$ Dept of Anesthesia, University of California San Francisco, San Francisco, CA, USA. ${ }^{5}$ Division of Pulmonary and Critical Care Medicine, Dept of Internal Medicine, University of Michigan Medical School, Ann Arbor, MI, USA. ${ }^{6}$ Dept of Microbiology and Immunology, University of Michigan Medical School, Ann Arbor, MI, USA. ${ }^{7}$ Michigan Center for Integrative Research in Critical Care, Ann Arbor, MI, USA.

Correspondence: Lieuwe D.J. Bos, Intensive Care, Amsterdam University Medical Centers, location AMC, University of Amsterdam, Infection and Immunity, Meibergdreef 9, G3-226, Amsterdam, 1105AZ, The Netherlands.

E-mail: 1.d.bos@amsterdamumc.nl

Received: 13 July 2020 | Accepted: 17 July 2020

Conflict of interest: L.D.J. Bos reports grants from the Dutch Lung Foundation (young investigator grant, Dirkje Postma Award and a public-private partnership grant), and personal fees from Bayer (for consultancy), outside the submitted work. P. Sinha has nothing to disclose. R.P. Dickson has nothing to disclose. 


\section{References}

1 Bhatraju PK, Ghassemieh BJ, Nichols M, et al. COVID-19 in critically ill patients in the Seattle region - case series. N Engl J Med 2020; 382: 2012-2022.

2 Bos LDJ, Paulus F, Vlaar APJ, et al. Subphenotyping ARDS in COVID-19 patients: consequences for ventilator management. Ann Am Thorac Soc 2020; in press [https://doi.org/10.1513/AnnalsATS.202004-376RL].

3 Ziehr DR, Alladina J, Petri CR, et al. Respiratory pathophysiology of mechanically ventilated patients with COVID-19: a cohort study. Am J Respir Crit Care Med 2020; 201: 1560-1564.

4 Auld SC, Caridi-Scheible M, Blum JM, et al. ICU and ventilator mortality among critically ill adults with coronavirus disease 2019. Crit Care Med 2020; in press [https://doi.org/10.1097/CCM.0000000000004457].

5 Cummings MJ, Baldwin MR, Abrams D, et al. Epidemiology, clinical course, and outcomes of critically ill adults with COVID-19 in New York City: a prospective cohort study. Lancet 2020; 395: 1763-1770.

6 Schenck EJ, Hoffman K, Goyal P, et al. Respiratory mechanics and gas exchange in COVID-19 associated respiratory failure. Ann Am Thorac Soc 2020; in press [https://doi.org/10.1513/AnnalsATS.202005-427RL].

7 Haudebourg AF, Perier F, Tuffet S, et al. Respiratory mechanics of COVID-19- versus non-COVID-19-associated acute respiratory distress syndrome. Am J Respir Crit Care Med 2020; 202: 287-290.

8 Gattinoni L, Coppola S, Cressoni M, et al. COVID-19 does not lead to a "typical" acute respiratory distress syndrome. Am J Respir Crit Care Med 2020; 201: 1299-1300.

9 Mauri T, Spinelli E, Scotti E, et al. Potential for lung recruitment and ventilation-perfusion mismatch in patients with the acute respiratory distress syndrome from coronavirus disease 2019. Crit Care Med 2020; 48: 1129-1134.

10 Rello J, Storti E, Belliato M, et al. Clinical phenotypes of SARS-CoV-2: implications for clinicians and researchers. Eur Respir J 2020; 55: 2001028.

11 Galetke W, Feier C, Muth T, et al. Reference values for dynamic and static pulmonary compliance in men. Respir Med 2007; 101: 1783-1789.

12 Dohi S, Gold MI. Pulmonary mechanics during general anaesthesia. The influence of mechanical irritation on the airway. Br J Anaesth 1979; 51: 205-214.

13 ARDS Definition Task Force, Ranieri VM, Rubenfeld GD, et al. Acute respiratory distress syndrome: the Berlin Definition. JAMA 2012; 307: 2526-2533.

14 Gattinoni L, Coppola S, Cressoni M, et al. Reply to: Hedenstierna et al, Haouzi et al, Maley et al, Fowler et al, Bhatia and Mohammed, Bos, \& Koumbourlis and Motoyama. Am J Respir Crit Care Med 2020; in press [https:/ doi.org/10.1164/rccm.202004-1052LE].

15 Bos LDJ, Sinha P, Dickson RP. The perils of premature phenotyping in COVID-19: a call for caution. Eur Respir J 2020; 56: 2001768

16 Schaller T, Hirschbühl K, Burkhardt K, et al. Postmortem examination of patients with COVID-19. JAMA 2020; 323: 2518-2520.

17 Ackermann M, Verleden SE, Kuehnel M, et al. Pulmonary vascular endothelialitis, thrombosis, and angiogenesis in COVID-19. N Engl J Med 2020; 383: 120-128.

18 Alhazzani W, Møller MH, Arabi YM, et al. Surviving Sepsis Campaign: guidelines on the management of critically ill adults with coronavirus disease 2019 (COVID-19). Crit Care Med 2020; 48: e440-e469.

19 Bos LDJ. COVID-19 related acute respiratory distress syndrome: not so atypical. Am J Respir Crit Care Med 2020; in press [https://doi.org/10.1164/rccm.202004-1423LE].

20 Sinha P, Matthay MA, Calfee CS. Is a "cytokine storm" relevant to COVID-19? JAMA Intern Med 2020; in press [https://doi.org/10.1001/jamainternmed.2020.3313].

21 Gattinoni L, Chiumello D, Caironi P, et al. COVID-19 pneumonia: different respiratory treatments for different phenotypes? Intensive Care Med 2020; 46: 1099-1102.

22 Calfee CS, Delucchi K, Parsons PE, et al. Subphenotypes in acute respiratory distress syndrome: latent class analysis of data from two randomised controlled trials. Lancet Respir Med 2014; 2: 611-620.

23 Famous KR, Delucchi K, Ware LB, et al. Acute respiratory distress syndrome subphenotypes respond differently to randomized fluid management strategy. Am J Respir Crit Care Med 2017; 195: 331-338.

24 Bos LD, Schouten LR, van Vught LA, et al. Identification and validation of distinct biological phenotypes in patients with acute respiratory distress syndrome by cluster analysis. Thorax 2017; 72: 876-883.

25 Delucchi K, Famous KR, Ware LB, et al. Stability of ARDS subphenotypes over time in two randomised controlled trials. Thorax 2018; 73: 439-445.

26 Prescott HC, Calfee CS, Thompson BT, et al. Toward smarter lumping and smarter splitting: rethinking strategies for sepsis and acute respiratory distress syndrome clinical trial design. Am J Respir Crit Care Med 2016; 194: $147-155$

27 Marini JJ, Gattinoni L. Management of COVID-19 respiratory distress. JAMA 2020; 323: 2329-2330.

28 Fan E, Beitler JR, Brochard L, et al. COVID-19-associated acute respiratory distress syndrome: is a different approach to management warranted? Lancet Respir Med 2020; 8: 816-821.

29 Tobin MJ, Laghi F, Jubran A. Caution about early intubation and mechanical ventilation in COVID-19. Ann Intensive Care 2020; 10: 78. 